\title{
O arco aórtico do sauim (Saguinus niger) e sua importância no processo de urbanização
}

\author{
Érika Branco ${ }^{1 *}$ \\ Mariana Barbara Cruz de Aragão ${ }^{1}$ \\ Danielli Martinelli Martins ${ }^{1}$ \\ Daiene Costa do Carmo ${ }^{2}$ \\ Juliana Teixeira Santos ${ }^{2}$ \\ Ana Rita de Lima ${ }^{1}$

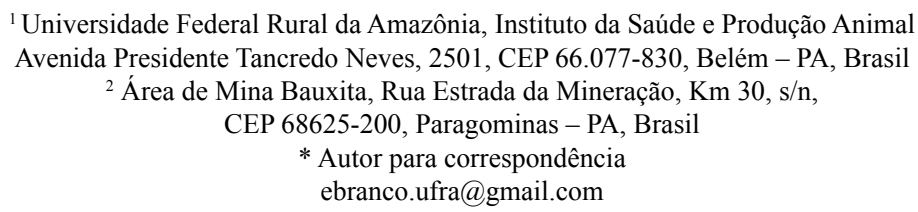

Submetido em 04/05/2017

Aceito para Publicação em 07/08/2017

\section{Resumo}

Distribuído geograficamente em todos os estados da Federação do Brasil, o sauim está presente em grandes centros urbanos devido ao aumento quantitativo desta espécie, e, tendo em vista que a prática médica e cirúrgica de animais selvagens está mais presente na medicina veterinária, visamos colaborar com o conhecimento da anatomia do arco aórtico e seus ramos colaterais, contribuindo no contexto de emergências médicas. Foram estudados quatro animais, sendo que três indivíduos apresentaram arquitetura do arco aórtico similar ao descrito nos mamíferos domésticos, com presença da artéria subclávia esquerda e artéria braquiocefálica, e em um animal a disposição dos ramos colaterais do arco aórtico seguiu o padrão encontrado em humanos, com a presença da artéria braquiocefálica, artéria carótida comum esquerda e artéria subclávia esquerda. Independentemente da configuração inicial do arco aórtico do sauim, da artéria braquiocefálica surgiram sequencialmente as artérias subclávia direita, vertebral direita, torácica interna direita, costocervical direita e cervical superficial direita. A artéria subclávia esquerda originou os mesmos ramos correlatos com o antímero direito.

Palavra-chave: Angioarquitetura; Aorta; Coração; Vascularização arterial

\section{Abstract}

The aortic arch of the black tamarin (Saguinus niger) and its importance in relation to urbanization. The black tamarin (Saguinus niger) occurs in all states of Brazil, including large urban centers due to population growth. Considering that the practice of medicine and surgery on wild animals has become more prevalent in veterinary medicine, this work describes the aortic arch of $S$. niger with the goal of aiding emergency surgeries. Four animals were studied. Three individuals had an aortic arch like the arch described for domesticated 
animals, including the presence of a left subclavian artery and brachiocephalic artery. In one individual, the arrangement of the collateral branches of the aortic arch followed the pattern found in humans, including the presence of a brachiocephalic artery, left common carotid artery, and left subclavian artery. Regardless of the initial configuration of the aortic arch of the black tamarin, the right subclavian, right vertebral, right internal thoracic, right costocervical and right superficial cervical arteries arose, sequentially, from the brachiocephalic artery. The branches formed by the left subclavian artery correlated with the right antimer.

Key words: Angioarchitecture; Aorta; Heart; Arterial vascularization

\section{Introdução}

O processo de urbanização de animais selvagens se dá principalmente devido ao acesso a estradas e ferrovias, uma vez que muitos espécimes são atraídos para essas áreas seja para auxiliar no processo de termorregulação, para simples deslocamento, para acessar grãos e outros alimentos deixados por veículos ou então para fins de acesso a carcaças de outros animais. Todos esses motivos acabam expondo muitos indivíduos da fauna silvestre brasileira ao risco de atropelamento (ERRITZOE et al., 2003; BAGATINI, 2006), porém, nem sempre o médico veterinário consegue intervir nessas situações e auxiliar no salvamento da vida desses animais.

Por outro lado, a prática da clínica médica e cirúrgica de animais selvagens está se tornando cada vez mais uma realidade notável, tendo em vista o constante aumento da população de animais selvagens domesticados e legalizados (SAITO et al., 2010). A elevada plasticidade ecológica e a aproximação com os seres humanos em busca de alimentos alternativos acabam sendo alguns dos fatores que favorecem essa coexistência, porém, alguns efeitos negativos desse processo de urbanização, como a agressividade, ocorrem por competição direta (SAITO et al., 2010), levando ao declínio da espécie, devido à pressão antrópica (MONTICELLI; MORAIS, 2015).

Para que os médicos veterinários possam desempenhar papel adequado frente às diferentes situações nesses pacientes, é necessário o conhecimento da anatomia e fisiologia desses indivíduos. Assim, conhecer o sistema circulatório como um todo e sua dinâmica é de grande valor para este cenário desafiador, sendo o sauim um pequeno primata neotropical, com notável presença em todos os estados da Federação, o que inclui a região Norte do Brasil (AURICCHIO, 1995); e, por transitarem nos centros urbanos, esses animais estão sujeitos a acidentes como pisoteios, eletrocussão e traumas vasculares.

Nesse contexto, devemos destacar a aorta como a principal artéria do corpo, e que emite ramos de extrema importância para os órgãos vitais, surgindo do ventrículo esquerdo, dirigindo-se cranialmente para a aorta ascendente e formando o arco aórtico, que ocupa o teto da cavidade torácica em direção ao abdome, passando a ser denominada caudalmente como aorta abdominal (SCHALLER, 1999).

Em humanos, o arco aórtico é considerado como uma estrutura de grande importância para os cirurgiões e hemodinamicistas, principalmente nos casos de aneurisma ou dissecação do arco aórtico, devido ao tratamento ser baseado em procedimento cirúrgico complexo. Desta forma, conhecer a anatomia do arco aórtico e de seus ramos pode ser o diferencial quando a questão é salvar uma vida (DEMERTZIS et al., 2010).

Nos animais domésticos, os ramos colaterais do arco aórtico já estão bem definidos, registrando a artéria braquiocefálica e a artéria subclávia esquerda, sendo a primeira responsável pela origem da artéria subclávia direita e das artérias carótidas comuns direita e esquerda (EVANS; LAHUNTA, 1994). No entanto, não há registro dessas informações em sauim, colocando em desvantagem o médico veterinário que atua na clínica médica e cirúrgica quanto ao sucesso terapêutico.

Diante do exposto, visamos suprir esta falta literária, uma vez que isso compromete possíveis abordagens cirúrgicas, gerando informações sobre os ramos colaterais do arco aórtico do sauim para orientar profissionais da área nos processos de abordagens mais invasivas. 


\section{Material e Métodos}

Foram avaliados quatro sauins, sendo três fêmeas e um macho, adultos jovens, oriundos da Mina Bauxita Paragominas, Pará, Brasil/Hydro Paragominas, sob autorização SEMA-PA no 55/2009 e 522/2009, que, após morte por atropelamento, foram congelados e enviados ao Laboratório de Pesquisa Morfológica Animal (LaPMA), da Universidade Federal Rural da Amazônia (UFRA).

Os animais foram descongelados em água corrente por período médio de $2 \mathrm{~h}$, e então foi acessada a aorta abdominal por laparotomia, a fim de realizar perfusão de látex Neoprene 650.

Após evidenciação do sistema arterial, os cadáveres foram fixados com solução de formoldeído a $10 \%$ por infusão intramuscular, intracavitária e subcutânea, mantendo-os submersos na mesma solução fixadora por no mínimo sete dias.

A nomenclatura anatômica utilizada foi baseada na Nomina Anatômica Veterinária (INTERNATIONAL COMMITTEE ON VETERINARY GROSS ANATOMICAL NOMENCLATURE, 2012).

\section{Resultados}

Os animais investigados apresentaram dois arranjos diferentes quanto à disposição de alguns ramos colaterais do arco aórtico, no entanto, as artérias relacionadas com o antímero direito do tórax comportaram-se de forma igual em todos os indivíduos, isto é, emergindo do arco aórtico, diretamente da artéria braquiocefálica passando obliquamente para a direita da superfície ventral da traqueia. Esse tronco contribuiu para formação das artérias carótida comum direita e subclávia direita, o que levou à formação das artérias vertebral direita, torácica interna direita, costocervical direita e cervical superficial direita. Já no espaço axilar, a artéria subclávia direita originou a artéria axilar direita (Figura 1 e 2).

Os ramos colaterais do arco aórtico relacionados com o antímero torácico esquerdo apresentaram-se de forma diferente em uma das fêmeas de sauim, na qual do referido arco surgiram mais dois ramos, formando artéria carótida comum esquerda, que era ventral ao esôfago, e, paralelamente, artéria subclávia esquerda, totalizando, então, três ramos arteriais oriundos do arco aórtico (Figura 1B).

FIGURA 1: (A - B): Fotomacrografia do arco aórtico do sauim. Arco aórtico (1), artéria braquiocefálica (2), artéria subclávia direita (3), artéria subclávia esquerda (4), artéria carótida comum direita (5), artéria carótida comum esquerda (6), artéria torácica interna direita (7), artéria torácica interna esquerda (8), artéria vertebral direita (9), artéria vertebral esquerda (10), artéria costocervical direita (11), artéria costocervical esquerda (12), artéria cervical superficial direita (13), artéria cervical superficial esquerda (14), artéria axilar direita (15) e artéria axilar esquerda (16). A artéria vertebral esquerda passa dorsalmente pela artéria carótida comum esquerda, sendo de difícil visualização (seta).
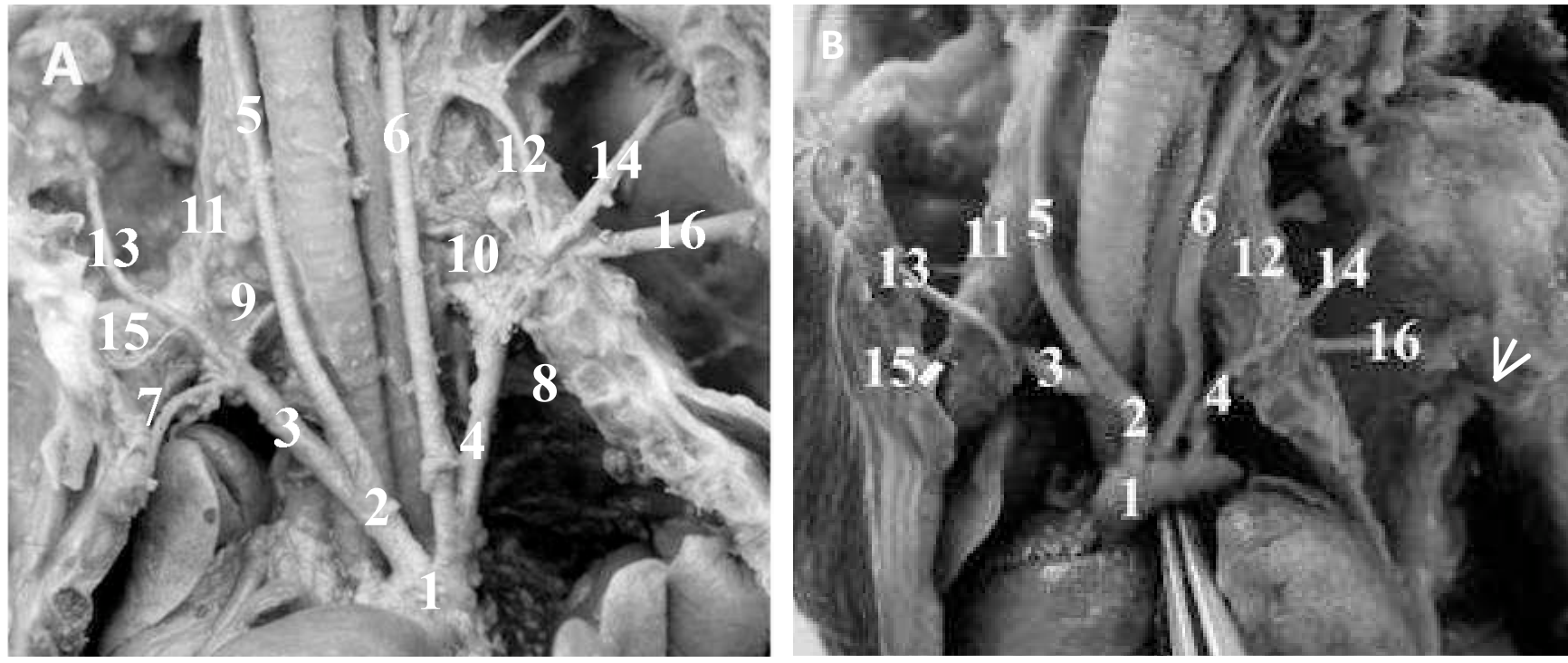
FIGURA 2: Esquematização do arco aórtico do sauim. (A): Arco aórtico (1), artéria subclávia esquerda (2), artéria carótida comum esquerda (3), artéria carótida comum direita (4), artéria braquiocefálica (5), artéria subclávia direita (6), artéria torácica interna direita (7), artéria vertebral direita (8), artéria axilar esquerda (9), artéria axilar direita (10), artéria cervical superficial esquerda (11), artéria cervical superficial direita (12), artéria costocervical esquerda (13) e artéria costocervical direita (14). (B): Arco aórtico (1), artéria subclávia esquerda (2), artéria carótida comum esquerda (3), artéria carótida comum direita (4), artéria braquiocefálica (5), artéria subclávia direita (6), artéria torácica interna esquerda (7), artéria torácica interna direita (8), artéria vertebral esquerda (9), artéria vertebral direita (10), artéria axilar esquerda (11), artéria axilar direita (12), artéria cervical superficial esquerda (13), artéria cervical superficial direita (14) e artéria costocervical esquerda (15).
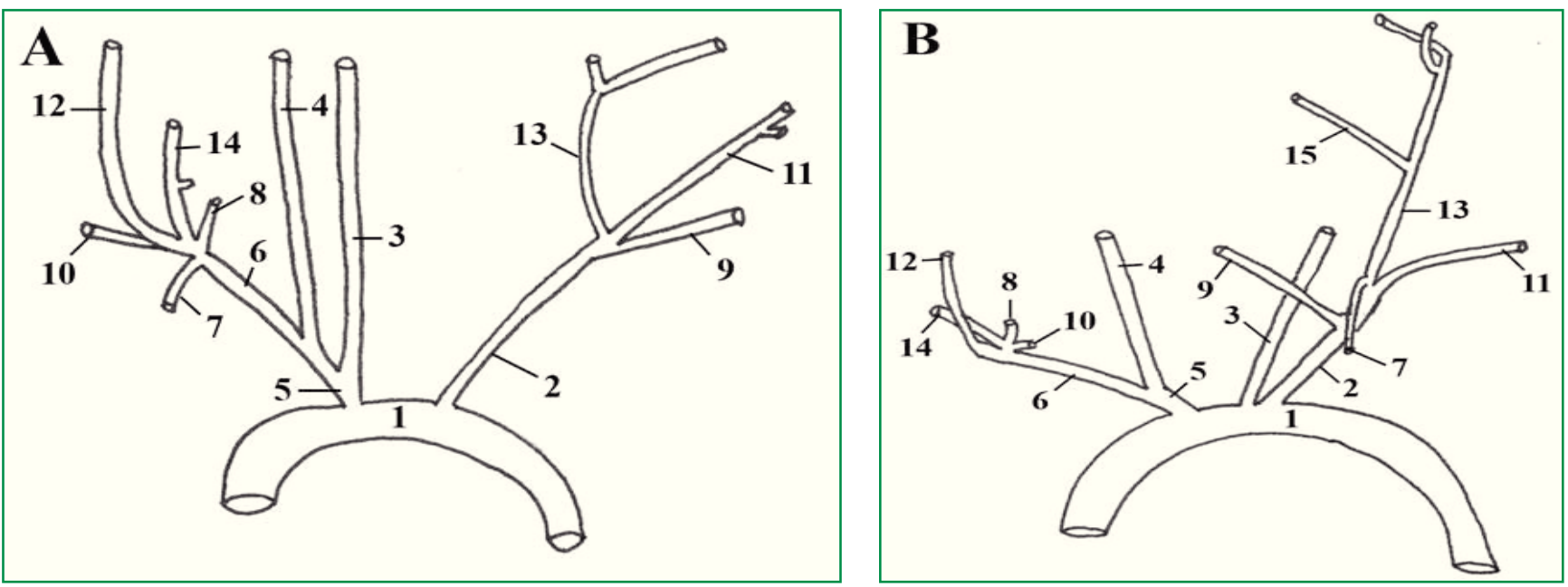

Para maior compreensão da disposição dos vasos evidenciados, optamos pela esquematização de cada artéria, conforme observado na Figura 2.

Da artéria subclávia esquerda surgiram ainda: artéria costocervical esquerda, artéria cervical superficial esquerda e artéria axilar esquerda. Diferentemente da direita, a artéria vertebral esquerda, neste exemplar, era de difícil visualização, direcionando-se ligeiramente pela face dorsal da artéria carótida comum esquerda (Figura 1A e 2A).

Nos outros animais, a artéria carótida comum esquerda e a artéria subclávia esquerda apareceram separadamente, no entanto, ambas a partir do arco aórtico (Figura 1A e 2A).

\section{Discussão}

Os primatas não humanos em geral têm grande importância no universo científico, levando ao aumento significativo de seu uso no contexto da pesquisa, devido à semelhança anatômica e comportamental em relação ao homem, tornando-os modelos experimentais (SOUZA, 2013). No entanto, a literatura relevante para o tema aqui proposto é totalmente rara, dificultando o processo de comparação.

O gênero Saguinus é um dos mais estudados como modelo experimental de lesões arteriais (FERRARI; MARTINS, 1992), todavia, não há registros de sua angioarquitetura, portanto, a abordagem cardiovascular destes indivíduos avança para uma situação de trauma.

Considerando a presença constante de algumas espécies de primatas não humanos em grandes centros urbanos, sujeitos a diversas lesões (MENDES, 1989; MONTICELLI; MORAIS, 2015), e a substancial e crescente atividade de médicos veterinários que trabalham diretamente com práticas médicas e cirúrgicas de animais selvagens, compreender a anatomia desses indivíduos é de fundamental importância para que suas performances sejam precisas, melhorando a expectativa de vida.

Entre os traumas avaliados em primatas não humanos, destaca-se o acometimento do sistema cardiovascular, principalmente por atropelamento e queda, uma vez que, de acordo com Bager et al. (2016), no Brasil os atropelamentos afetam mais de 475 milhões de vertebrados todos os anos, ou 15 animais a cada 
segundo; e sendo o sauim uma espécie muito comum em centros urbanos, torna-se importante o estudo da anatomia de seu sistema vascular, levando-nos mais precisamente a abordar os ramos colaterais do arco aórtico.

A literatura clássica sobre a anatomia do arco aórtico nos seres humanos revela que há variações anatômicas de ramos deste arco, podendo surgir cinco tipos (A, B, C, D e E), sendo o tipo A o mais encontrado, composto pela artéria braquiocefálica, seguido pela artéria carótida comum esquerda e pela artéria subclávia esquerda. O tipo B contempla a artéria carótida comum esquerda, ao longo da artéria braquiocefálicas, enquanto que o tipo $\mathrm{C}$ é constituído por parte da artéria carótida comum esquerda e da artéria braquiocefálica, juntamente com a artéria subclávia direita e a artéria carótida comum direita. O tipo D apresenta alteração expressiva devido à ausência da artéria braquiocefálica, de modo que as duas artérias carótidas e as duas subclávias (direita e esquerda) surgem diretamente do arco aórtico. Por fim, no tipo E há a presença de duas artérias braquiocefálica, de modo que a artéria subclávia esquerda e a artéria carótida comum esquerda surgem do segundo ramo da artéria braquiocefálica (SILVA et al., 2012; PUTZ; PABST, 2013).

Esta variação de tipos de arranjos de ramos colaterais do arco aórtico nos leva a uma importante descoberta e reflexão, pois segundo Silva et al. (2012), o tipo A é o mais comumente encontrado em seres humanos, e o tipo E não foi registrado em um total de 19 cadáveres por eles avaliados, diferindo de nossos achados, uma vez que em uma amostra expressivamente menor, totalizando quatro cadáveres de sauim, três apresentavam arco aórtico tipo A e um tipo E. Esse resultado corrobora o descrito por Moore e Dalley (2014), que também descrevem a presença do tipo E em seu trabalho voltado para a prática clínica em humanos.

Apesar de nossos achados em sauim, a literatura revela que há variação também entre outras espécies de primatas não humanos, como é o caso do o sagui-detufo-preto (Callithrix penicillata) (LIMA et al., 2014), que apresenta uma angioarquitetura do arco aórtico mais semelhante à descrita em mamíferos domésticos, ou seja, a artéria braquiocefálica emite a artéria subclávia direita e o tronco bicarotídeo, com a artéria subclávia esquerda partindo diretamente do arco aórtico (GHOSHAL, GETTY, 1986; EVANS; LAHUNTA, 1994; DYCE et al., 2010), sendo então classificado como tipo C (PUTZ; PABST, 2013).

Dados como os relatados aqui tornam-se valiosos para o âmbito clínico e cirúrgico, pois podem salvaguardar a integridade dos ramos colaterais do arco aórtico quando forem necessárias intervenções técnicas na face ventral do pescoço (SILVA et al., 2012).

Após nossas descobertas, é surpreendente como extrapolar o conhecimento da anatomia humana e da anatomia dos animais domésticos em geral para primatas não humanos tende a demonstrar o risco grave à saúde desses indivíduos, quando for necessário realizar abordagens de emergência clínica e cirúrgica. Assim, conhecer a morfologia de várias ordens pode ser o diferencial no sucesso da conservação das espécies de animais selvagens, que cada vez mais se aproximam do homem em busca de alimentos.

\section{Referências}

AURICCHIO, P. Primatas do Brasil. São Paulo: Terra Brasilis, 1995. 168 p.

BAGATINI, T. Evolução dos índices de atropelamento de vertebrados silvestres nas rodovias do entorno da Estação Ecológica Águas Emendadas, DF/Brasil e eficácia de medidas mitigadoras. 2006. 78 f. Dissertação (Mestrado em Ecologia) Universidade de Brasília, Brasília. 2006.

BAGER, A.; LUCAS, P. S.; BOURSCHEIT, A.; KUCZACH, A.; MAIA, B. Os caminhos da conservação da biodiversidade brasileira frente aos impactos da infraestrutura viária. Biodiversidade Brasileira, Brasília, v. 6, p. 75-86, 2016.

DEMERTZIS, S.; HURNI, S.; STALDER, M.; GAHL, B.; HERRMANN, G.; VAN DEN BERG, J. Aortic arch morphometry in living humans. Journal of Anatomy, London, v. 217, n. 5, p. 588-596, 2010.

DYCE, K. M.; SACK, W. O.; WENSING, C. J. Tratado de anatomia veterinária. Rio de Janeiro: Guanabara Koogan, 2010. $856 \mathrm{p}$.

ERRITZOE, J.; MAZGAJSKI, T. D.; REJT, L. Bird casualties on European roads - a review. Acta Ornithologica, Varsóvia, v. 38, p. 77-93, 2003.

EVANS, H. E.; LAHUNTA, A. D. E. Guia para a dissecação do cão. Rio de Janeiro: Guanabara Koogan, 1994. 206 p.

FERRARI, S. F.; MARTINS, E. S. Gummivory and gut morphology in two sympatric callitrichids (Callithrix emiliae and Saguinus fuscicollis weddelli) from Western Brazilian Amazonia. American 
Journal of Physical Anthropology, Cambridge, v. 88, p. 97-1103, 1992.

GHOSHAL, N. G.; GETTY, R. Anatomia dos animais domésticos. Rio de Janeiro: Guanabara Koogan, 1986. 1134 p.

INTERNATIONAL COMMITTEE ON VETERINARY GROSS ANATOMICAL NOMENCLATURE. Nomina Anatomica Veterinaria. Hannover: Editorial Committee, 2012. 177 p.

LIMA, A. R.; BRANCO, E.; NETO, J. R. S. Ramos colaterais do arco aórtico do Callithrix penicillata (saguí-de-tufo-preto). In: CONGRESSO BRASILEIRO DE MEDICINA VETERINÁRIA, 41, 2014, Gramado. Resumo... Gramado: CONBRAVET, 2014. Versão eletrônica.

MENDES, S. L. Estudo ecológico de Alouatta fusca (Primates: Cebidae) na Estação Biológica de Caratinga, MG. Revista Nordestina de Biologia, João Pessoa, v. 6, p. 71-104, 1989.

MONTICELLI, C.; MORAIS, L. H. Impactos antrópicos sobre uma população de Alouatta clamitans (Cabrera, 1940) em um fragmento de Mata Atlântica no estado de São Paulo: apontamento de medidas mitigatórias. Revista Biociências, Taubaté, v. 21, p. 14-26. 2015.
MOORE, K. L.; DALLEY, A. F. Anatomia orientada para a clínica. Rio de Janeiro: Guanabara Koogan, 2014. 1136 p.

PUTZ, R.; PABST, R. Sobotta: atlas de anatomia humana. Rio de Janeiro: Guanabara Koogan, 2013. 1200 p.

SAITO, C. H.; BRASILEIRO, L.; ALMEIDA, L. E.; TAVARES, M. C. H. Conflitos entre macacos prego e visitantes no Parque Nacional de Brasília: possíveis soluções. Sociedade \& Natureza, Uberlândia, v. 22, p. 515-524, 2010.

SCHALLER, O. Nomenclatura anatômica veterinária ilustrada. São Paulo: Manole, 1999. 613 p.

SILVA, K. F.; PEREIRA, K. F.; ALBUQUERQUE, K. P.; TEIXEIRA, C. S.; ODA, J. Y. Estudo descritivo das variações anatômicas dos ramos do arco aórtico. Arquivos de Ciências da Saúde da UNIPAR, Umuarama, v. 16, p. 101-103, 2012.

SOUZA, A. C. Avaliação ultrassonográfica abdominal e descrição de parâmetros normais em primatas não humanos. 2013. 104 f. Dissertação (Mestrado em Ciências Veterinárias) Universidade Federal do Paraná, Curitiba. 2013. 